\title{
Recuperación natural y recuperación con tratamiento del consumo de drogas y alcohol
}

\author{
Augusto Pérez Gómez*; Diana Raquel Sierra Acuña** \\ *Corporación Nuevos Rumbos y Robert Wood Johnson Medical School, New Jersey. \\ **Corporación Nuevos Rumbos. \\ Enviar correspondencia a:
}

Augusto Pérez Gómez. Corporación Nuevos Rumbos. Calle 108A \# 4-15, Bogotá, D.C. Colombia. Correo electrónico aperez@nuevosrumbos.org.

Recibido: Febrero de 2007 Aceptado: Julio de 2007

\section{RESUMEN}

La presente investigación examina el concepto de recuperación natural de los problemas derivados del consumo de alcohol, marihuana, cocaína y heroína, cada una sola o en cualquier combinación. Se trabajó con dos grupos de sexo masculino y dos de sexo femenino (40 hombres entre 18 y 60 años, y 19 mujeres entre 18 y 55 años) de la ciudad de Bogotá que tenían por lo menos un año de abstinencia. Se analizaron principalmente las razones para entrar a tratamiento o para suspender el consumo sin acudir a éste, los factores asociados al mantenimiento de la abstinencia y las dificultades y las amenazas de la misma. Se encontraron algunas diferencias significativas entre los grupos con y sin tratamiento, y también entre los hombres y las mujeres, especialmente en lo que se refiere a los factores asociados al mantenimiento de la recuperación. Sin embargo en ambos casos los vínculos familiares y afectivos -el 'capital social'- aparecen como el factor de mayor importancia en la decisión de parar el consumo en todos los grupos. Por otra parte, el compromiso con las metas y el proyecto de vida personal son los motivos que estimulan el mantenimiento de la abstención o del consumo moderado, elementos que evidencian el paso progresivo de procesos cognitivos y emocionales con referentes externos a procesos en los que se privilegian los referentes de tipo interno y asociados al logro personal.

Palabras clave: recuperación natural, dependencia a las drogas, farmacodependencia, tratamiento de drogadicción.

\section{ABSTRACT}

This study examines the concept of natural recovery (without formal treatment) from problems associated with alcohol, marijuana, cocaine and heroin abuse, each one alone or in any combination. Two groups of males ( $40 \mathrm{Ss}$ between 18 and 60 years of age) and two groups of females ( 19 Ss between 18 and 55 years of age) with at least one year of abstinence were studied. The main issues considered were: reason for attending treatment or ceasing the use of substances, factors related to maintenance of abstinence, and difficulties and threats associated with abstinence. Several significant differences were found between groups with and without treatment, as well as between males and females, particularly regarding factors related to the maintenance of abstinence. In both cases family and affective links appear as the most relevant factors in the decision to stop using substances. On the other hand, commitment to one's goals and life project are the principal motives for maintaining abstinence or moderate consumption. This reflects the progressive transition from cognitive and emotional processes with external referents to processes with internal referents, associated with personal achievement.

Key words: Natural recovery, self-care rehabilitation, drug dependence, substance dependence, treatment of drug addiction.

\section{INTRODUCCIÓN}

$\mathbf{E}$

I consumo problemático y el abuso del alcohol y otras sustancias psicoactivas afectan la salud física y mental de los individuos, e interfieren con las responsabilidades y actividades familiares, sociales y laborales. Quien presenta un consumo problemático o abusivo de sustancias está afectando negativamente su vida y también (en la mayoría de las ocasiones) la de quienes lo rodean; sin embargo de estas personas, algunas continúan el consumo, mientras que otras lo dejan o lo moderan hasta evitar la problemática concomitante en el que ya no se presentan consecuencias negativas relacionadas.

No todos los individuos que han superado su problemática de consumo se han sometido a un tra- 
tamiento terapéutico formal para conseguir la recuperación (recurso largamente pensado y utilizado), sino que en muchos casos se trata de un proceso 'espontáneo', una recuperación 'natural'. Si bien el conocimiento respecto a los mecanismos que explican la compulsión al consumo se ha consolidado, aquellos que explican el proceso de recuperación natural se han estudiado en menor proporción. Además, existe un fuerte prejuicio avalado por reconocidas autoridades en el tema del tratamiento de alcohólicos y consumidores de sustancias ilegales, según el cual nadie se cura sin algún tipo de tratamiento estructurado para problemas de adicción (Burman, 1997).

Aun cuando ha sido utilizado en la literatura psicológica y especialmente en la psicoterapéutica, el término recuperación natural o espontánea es poco familiar para muchas personas. La intuición y el sentido común tienden a hacer pensar que hace referencia a una recuperación sin intervención de una causa exterior, que se da de forma automática o involuntaria. Así, uno de los mayores inconvenientes del término es que varias personas, incluidos profesionales de amplia experiencia, asumen esa palabra como si hiciera referencia a un milagro que no permite seguimiento o desglosamiento alguno y por ello no podría ser medido, estudiado o analizado seriamente a través de la investigación científica. Bergin (1971) argumentó que el término ha retardado el proceso científico porque lleva a pensar que los procesos de recuperación espontánea equivalen a no saber qué está pasando y la idea de que hay eventos que ocurren sin razón aparente es algo que el espíritu científico considera inaceptable.

Sin embargo, al momento actual son diversos los estudios que evidencian la existencia y complejidad de la recuperación natural (Klingemann, 1991, Sobell, 1992, Grandfiel y Cloud, 1996, Cunningham, 1999). Así por ejemplo en el Epidemiologic Catchment Area (ECA) realizado en Estados Unidos en el año 1991, se encontraron altos índices de recuperación natural en desórdenes por uso de sustancias (54\%-59\%). En este estudio se encontró incluso que el nivel de búsqueda de tratamiento para algunos desordenes psiquiátricos como los relacionados con el uso de SPA eran menores (24\%) en relación a los encontrados con desordenes como la depresión (54\%), los desordenes bipolares (61\%) y las somatizaciones (70\%). En otras palabras para algunos desórdenes, como el consumo de sustancias psicoactivas (SPA), muchos de aquellos que se recuperan lo hacen sin ningún tratamiento formal (Slutske, 2006).

Para el caso de América Latina, no encontramos estudios que busquen la comprensión de la recuperación natural, y mucho menos que estudien la existencia de este fenómeno en grupos particulares como la población femenina. Destacando la importancia de estudiar este fenómeno en nuestro medio, en esta investigación se realiza un primer análisis de los eventos y / o procesos cognitivos que operan o influyen en la recuperación natural versus recuperación bajo tratamiento considerando género. Se exploran diferencias respecto a razones que llevan a la recuperación, factores que contribuyen al mantenimiento de la recuperación, y motivos para no recibir tratamiento.

\section{MÉTODO}

\section{Participantes}

La muestra estuvo conformada por 40 hombres entre 18 y 60 años (promedio: 40 años), y 19 mujeres entre 18 y 55 años (promedio: 38 años), con un nivel educativo relativamente elevado (más de la mitad tanto de los hombres como de las mujeres tenían estudios universitarios); todos vivían en la ciudad de Bogotá, habían cumplido en un momento determinado los criterios de dependencia o consumo problemático del DSM IV a alcohol, cocaína, marihuana y / o heroína (solas o en cualquier combinación) y tenían al menos un año de recuperación o abstinencia. Las personas que participaron en el estudio habían ingresado en centros de tratamiento de las ciudades de Bogotá, Cali y Medellín, conocidos y referidos del equipo principal de investigación (estrategia de bola de nieve), y posteriormente personas desconocidas que contactaron al equipo investigador a través de las convocatorias hechas por medio impreso o Internet.

Definición de los grupos: (1) Grupo de recuperación natural o sin tratamiento formal (hombres: $n=11$; mujeres: $n=8)$ : sujetos con mejorías en desórdenes de diferentes tipos de adicciones que se dieron aún con la ausencia de cualquier tipo de tratamiento formal. No podían haber recibido consejo del médico de cabecera, o haber leído libros de auto ayuda para este fin. Podían haber asistido a menos de tres sesiones de Alcohólicos Anónimos (A.A). No se excluyeron de este grupo sujetos que pudieran haber recibido consejo de personas sin formación terapéutica, tales como sacerdotes, abogados, parapsicólogos, amigos, etc. (2) Grupo de Tratamiento formal o grupo de autoayuda con éxito. Sujetos que hubieran asistido a intervención formal, donde el objetivo principal fuera ayudar a personas que tienen problemas de consumo de alcohol u otras drogas. Incluye sujetos que han usado fármacos semejantes al Disulfiram, consultado con un médico que diagnostica la importancia de cambiar de comportamiento y que hayan leído bibliografía terapéutica. La finalización del tratamiento se debió dar como mínimo un año antes de la participación en el estudio (hombres: $n=29$; mujeres: $n=11$ ). 


\section{Instrumentos}

Se utilizaron cuatro instrumentos: Primero, Cuestionario de filtro control de recuperación, que permite rastrear si el sujeto está o no recuperado (Pérez 2003), con un total de 4 items; segundo, Cuestionario de datos demográficos que incluía 18 ítems dirigidos a identificar datos básicos del sujeto, así como su frecuencia de consumo, el tiempo de abstención y la presencia o ausencia de diversas intervenciones o tratamientos terapéuticos dirigidos al cambio del comportamiento de consumo (Pérez 2003); tercero, Entrevista Semiestructurada (Pérez 2003) que permite rastrear las razones que facilitaron la recuperación y su mantenimiento, las personas que más influyeron en dicha recuperación, las barreras ante el tratamiento formal y los motivos para abandonar el tratamiento (en caso de haberlo hecho), desarrollado con base en una entrevista utilizada en estudios previos de recuperación espontánea (Sobell, 2000), cuenta con 133 ítems específicos; y, finalmente una versión en español del AUDIT (Alcohol Use Disorders Identification Test), instrumento que permite rastrear problemas relacionados con el uso de alcohol, diagnosticando si había dependencia a las sustancias. EI AUDIT fue validado en los Estados Unidos con población de origen hispano, actualmente bilingüe; como se presenta una cierta contaminación idiomática inevitable, el instrumento fue corregido por uno de los autores de este artículo (Pérez, 2003). Se utilizó una versión del AUDIT reformada ligeramente por el equipo de Linda Sobell para incluir otras drogas.

\section{Procedimiento}

Las personas fueron contactadas, y los datos recolectados, entre enero y noviembre de 2005 en las ciudades de Bogotá, Medellín y Cali. Una vez realizado el contacto con los interesados, desde los diferentes contextos en que se ubicaron (centros de tratamiento, contacto con psicólogos, correo electrónico) se les invitó a una entrevista individual en un sitio adecuado para ello (consultorio psicológico). Se explicaron los objetivos de la investigación y su participación voluntaria, invitándolos a firmar los consentimientos informados.

Para la aplicación del instrumento, un profesional en psicología dio los instrumentos de manera individual a cada participante, quienes respondieron a la entrevista semiestructurada de manera voluntaria e individual, en un espacio de carácter privado y por un tiempo promedio de hora y media. Todas las personas aprobaron y firmaron el consentimiento informado de participación en la investigación.
Para efectos de definiciones, recuperación se entendió como un período de abstinencia total mínimo de un año (aplicable a todas las sustancias) o como un consumo de bajo riesgo según los criterios de la OMS siempre y cuando no le cause problemas laborales, médicos, sociales y/o familiares. 'Bajo riesgo' se definió de la siguiente manera: hombres 3 bebidas estándar o menos por día; mujeres: 2 bebidas estándar o menos por día; Bebidas estándar: Una botella de cerveza: $285 \mathrm{ml}$; Copa de vino: $120 \mathrm{ml}$; Copa bebida destiladas: $30 \mathrm{ml}$; Bebidas aperitivo como el Vermouth: $60 \mathrm{ml} ; 3$ vasos de Sidra: 285ml; Marihuana, hachís: 2 consumos o menos al año; Cocaína, heroína y otras drogas: 1 consumo al año como máximo (no inyectado).

\section{Análisis de datos}

Se realizó el procesamiento de los datos de la encuesta en el programa informático SPSS 10.0, con una significación de $p<0,5$; mientras que la información cualitativa se agrupó en categorías de análisis determinadas por la estructura de la entrevista utilizada para este fin.

\section{RESULTADOS}

\section{Respecto a las razones que llevaron a la recupe- ración}

Diferencias y similitudes encontradas entre los dos grupos bajo la condición de ser hombre

En el grupo con tratamiento se observó que las motivaciones más sobresalientes para tomar la decisión de recuperarse se centran en tener mucho que perder, especialmente en lo relacionados con el círculo afectivo inmediato: relaciones de pareja y relaciones familiares. Posteriormente aparecen razones como el temor a las consecuencias del consumo en sí mismo $\mathrm{y}$ en otros.

Se observó también, especialmente entre aquellos que asisten a grupos de apoyo (AA, NA), la adopción de una identidad de adictos, en la cual el hecho de estar "sobrio" o "limpio" es la prioridad, pues sin esta no se concibe la posibilidad de tener una vida, ni salud, ni familia, ni trabajo, lo cual hace referencia a la naturaleza de la enfermedad. Este motivo es vital en el mantenimiento de la recuperación.

El mantenimiento de la "sobriedad" o "limpieza", el mejoramiento de la salud y la recuperación física, se apoyan sobre los compromisos hechos consigo mismos, el soporte del poder superior y el miedo a perder lo logrado (familia y logros personales entre otros). 
El apoyo de una red social se convierte en un evento determinante en la recuperación de quienes asisten a tratamiento y lo han finalizado exitosamente. La familia se convierte no solo en un factor que motiva o presiona para la recuperación, sino también en motivador y apoyo en el mantenimiento de la misma.

Es interesante notar que el "estar involucrado en el proceso de recuperación de un adicto (familiar, amigo, pareja)" se convierte en un factor importante del mantenimiento de los logros en el grupo con tratamiento (el 72\% del grupo lo afirma), mientras que para el $91 \%$ del grupo sin tratamiento éste factor no tiene ninguna incidencia; lo notable en sí mismo es la importancia que le atribuyen los del primer grupo, entre quienes este tipo de comportamiento se promueve activamente; este no es, obviamente, el caso, en quienes se recuperan sin ayuda formal.

Las razones que motivan la suspensión del consumo en el grupo sin tratamiento se refieren generalmente al temor de perder la familia / pareja, y a deteriorar más las relaciones familiares (generar más conflictos): pero en el mantenimiento, prima tener presente los compromisos que se han pactado consigo mismos, pensar en lo mejor que se está sin consumir y desear seguir de esta manera, y el apoyo de la pareja.

En este grupo, a diferencia del primero, se observa que algunos procesos de tipo cognoscitivo juegan un papel relevante en el mantenimiento de la sobriedad dentro de un esquema de costo-beneficio (consumono consumo). Igualmente, se observan estrategias intencionadas de búsqueda de espacios gratificantes en los que no se utilicen la(s) sustancia(s)

\section{Dificultades al afrontar el tratamiento}

En el grupo que recibió tratamiento formal fueron básicamente las relacionadas con la aceptación (toma de conciencia) de la existencia de un problema en relación con el alcohol y/o las drogas, especialmente la aceptación de que en sus casos requerían de ayuda profesional para suspender el consumo y mantenerse abstemios; en este grupo la separación/distanciamiento de sus seres queridos fue otra de las dificultades afrontadas.

Por el contrario, para el grupo que nunca asistió a tratamiento las dificultades más grandes fueron las relacionadas con el cambio en las relaciones interpersonales y los ambientes claramente propiciadores del consumo. No se trataba tanto de una cuestión de aceptación del problema o de aceptación de ayuda, sino de evitación de riesgos.
Diferencias y similitudes encontradas entre los dos grupos bajo la condición de ser mujer

Las principales razones que reportaron las mujeres del grupo con tratamiento para suspender el consumo fueron en su orden: los conflictos que estaba generando el consumo en sus relaciones familiares, presencia de sentimientos de depresión e irritabilidad, y en menor grado de importancia aunque igualmente prevalentes: el distanciamiento notable de la familia (conflictos) y la afectación de su vida social y de las relaciones con sus amistades.

Por su parte en el grupo sin tratamiento, las razones que motivaron la suspensión del consumo fueron, en su orden: los conflictos generados en las relaciones familiares, darse cuenta de lo mal que estaban otras personas que consumían, el distanciamiento notable de la familia, notar las enfermedades que otros sufrían por el consumo. Las motivaciones emergentes para tomar la decisión de recuperarse se centran, al igual que en los grupos de hombres, en tener mucho que perder (especialmente en lo relacionados con el círculo afectivo familiar -conflictos-), pero se adiciona un elemento cognitivo: la conciencia en relación con el problema y sus consecuencias para sí mismas y para otros.

A diferencia del grupo con tratamiento, en el que se evidencia la influencia de los vínculos familiares como razones de suspensión de consumo, pero en el que no parece primar el temor a perderlos (quizás porque ya están muy deteriorados), en el grupo sin tratamiento son dos razones las que principalmente motivaron la suspensión del consumo: el temor a la perdida de la relación familiar o su empeoramiento, y la identificación con el sufrimiento de otros que consumían (o el temor de llegar a estar en su mismo estado), revelándose en este ultimo punto una clara actitud empática movilizadora de la recuperación.

\section{Diferencias y similitudes entre hombres y mujeres}

Entre las diferentes razones determinantes en la toma de decisión de suspender el consumo, no se encontraron diferencias significativas en ninguna de ellas. Se utilizo la prueba estadística chi-cuadrado.

\section{Respecto a factores que contribuyen a mantener la recuperación}

\section{Diferencias y similitudes entre tratados y no tratados}

Los factores que motivaron el mantenimiento de la recuperación en el grupo que se adhirió a un tratamiento fueron en su orden: tener presentes los compromisos hechos consigo mismas, pensar en lo mejor 
que se está y querer seguir así, haber aprendido a divertirse con actividades donde el consumo no existe, pensar en los problemas que generó el consumo en el pasado, asistir a grupos de AA y NA, sentir que sin consumir se puede mostrar la cara sin temor al juicio, pensar en que se es una adicta en recuperación.

Tres factores fueron los de mayor relevancia en el mantenimiento de la abstinencia en el caso del grupo sin tratamiento: tener presente los compromisos que se han pactado a sí mismos, aceptar que no hay ninguna razón válida para consumir y pensar en lo mejor que se está sin consumir y desear seguir de esta manera. El replanteamiento del proyecto de vida y el soporte de un "poder" espiritual fueron los factores que siguieron en importancia a los tres mencionados anteriormente.

Se evidencian procesos de tipo cognitivo similares en los dos grupos: mantener los compromisos hechos consigo mismas, mas que con familiares $u$ otros significativos, y el mantener el esquema beneficio-costo (no consumo-consumo) como elementos que refuerzan positivamente la idea de no consumir. De la misma manera se observan en los dos grupos estrategias intencionadas de espacios gratificantes en los que no se utilice la(s) sustancia(s): para el grupo que hizo tratamiento, estos espacios se incluyen en sus actividades terapéuticas (AA y NA), para quienes no asistieron a tratamiento otros espacios entran a cubrir la misma función.

\section{Diferencias y similitudes entre hombres y mujeres}

En el análisis inter-grupal realizado con la prueba estadística chi-cuadrado, se encontró que la razón "asistir a grupos A.A, N.A/ Auto-Ayuda con frecuencia", es uno de los factores que determinan diferencias en los grupos analizados; mientras que para el 85.7\% del grupo sin tratamiento este factor no ha tenido influencia en su mantenimiento, para el 100\% del grupo con tratamiento si lo ha tenido.

Asimismo, "pensar que soy un adicto en recuperación" es otra diferencia en la motivación al mantenimiento de la abstinencia o consumo moderado entre los dos grupos. Mientras para el $75 \%$ del grupo sin tratamiento no es una motivación para esto, para el $100 \%$ del grupo con tratamiento si es una motivación para el mantenimiento de su recuperación.

\section{Respecto a motivos para no recibir tratamiento formal}

\section{Dificultades y similitudes entre tratados y no tratados}

En el grupo que recibió tratamiento las mayores dificultades que se afrontaron al iniciar el mismo fueron básicamente las relacionadas con la aceptación (conciencia) de la existencia de un problema en relación con el alcohol y/o las drogas, especialmente de que en sus casos requerían de ayuda profesional para suspender el consumo y mantenerse abstemio; asimismo en este grupo la separación/distanciamiento de sus seres queridos fue otra de las dificultades afrontadas. Por el contrario para el grupo que nunca asistió a tratamiento, las dificultades más grandes fueron las relacionadas con el cambio en las relaciones interpersonales y los ambientes claramente propiciadores del consumo. No se trataba tanto de una cuestión de aceptación del problema o de aceptación de ayuda, sino de evitación de riesgos.

\section{Diferencias y similitudes entre hombres y mujeres}

Las diferencias encontradas entre los dos grupos se refieren, en principio, a razones propias de la participación del grupo de tratamiento en un espacio terapéutico (conciencia de ser "adicta", "necesidad de grupos terapéuticos"), y a dos elementos fundamentales: los sentimientos de depresión e irritabilidad -vivenciados por quienes realizaron tratamiento- con respecto a sí mismas, a su consumo y a las consecuencias de éste; mientras que el grupo de no tratamiento evidencia más una actitud empática y una identificación con los sentimientos dolorosos de otras personas que también se han encontrado en consumo. Estas diferencias no se encuentran entre los hombres. Sin embargo cabe destacar que razones fundamentales como los conflictos con familiares y el distanciamiento de ellos son elementos comunes en los dos grupos al momento de decidir dejar el consumo.

En relación a las razones para mantener la abstención o el consumo moderado, los dos grupos presentan mayores semejanzas que en el punto anterior: siendo el nivel de conciencia del problema la razón principal para querer continuar en recuperación; se observa, en ambos grupos, que algunos procesos de tipo cognoscitivo juegan un papel relevante en el mantenimiento de la sobriedad dentro de un esquema de costo-beneficio (consumo-no consumo).

\section{DISCUSIÓN}

Los resultados de este trabajo muestran que uno de los factores determinantes en la recuperación, con y sin tratamiento formal, es el apoyo de parte de la familia o amigos, tal y como lo planteaban Grandfiel y Cloud (1996) al destacar el "capital social" como factor esencial en los procesos de recuperación natural. Acorde a los planteamientos de Waldorf, Reinarman y Murphy (1991), las personas que poseen una red de 
apoyo (familiares, amigos, trabajo) parecen salir más fácilmente y con menos ayudas profesionales del consumo. El contexto social ejerce una gran influencia en la superación del abuso de alcohol y drogas. Para la mayor parte de los sujetos de este estudio, el "recibir apoyo de mi familia" es un factor que se asocia con la condición de haberse recuperado con o sin tratamiento de una manera significativa; aun así, debe resaltarse que hay diferencias significativas entre los dos grupos: el grupo con tratamiento lo percibe, en mayor medida, como un factor importante en comparación con la percepción de aquellos que no hicieron tratamiento formal.

La recuperación natural es un proceso complejo en el que intervienen múltiples factores que, al ser identificados, pueden ayudar a diseñar estrategias individuales y grupales para solucionar el problema del consumo abusivo de sustancias. Aparte de los beneficios derivados de la decisión del individuo (recuperarse, superar su problemática), hay un avance en la determinación de un modelo que se basa en la creencia sobre la capacidad de la persona para conseguir positivamente los resultados. Surge un énfasis en los méritos individuales, las fortalezas, la iniciativa para promover la responsabilidad en el cambio, en contraste directo con el modelo tradicional basado en la falta de poder sobre el control de sí mismo.

El temor a perder los vínculos familiares o con seres queridos, o que su deterioro progrese es para hombres y mujeres, sin distinción de tipo de tratamiento recibido (o no tratamiento) un elemento vital en la decisión de dejar o moderar el consumo de sustancias psicoactivas. La existencia de una red de apoyo social sería entonces un elemento de mejor pronóstico en los casos de recuperación de consumo de drogas. Para el caso de las mujeres, en comparación al grupo de hombres, se adicionan dos elementos en este proceso de toma de decisiones: por un lado, para el grupo que hizo un tratamiento formal, la percepción y validación de sentimientos de depresión e irritabilidad en sí mismas (asociados al consumo) se adiciona a las razones que se tuvieron en cuenta para dejar el consumo; mientras que para el grupo que se recuperó de manera "espontánea", es el "darse cuenta de" lo mal que estaban otros en consumo (evidente proceso cognitivo que tiene como referencia a otros diferentes al sí mismo).

Para este último grupo en particular, pareciera existir un elemento generador de reflexión referido a sí mismas y sus pérdidas, y otro que trasciende el sí mismo y les posibilita la identificación con otros como medio de análisis del si mismo.

En todos los grupos analizados el compromiso con las metas y el proyecto de vida personal (proyecto sin consumo) es el motivo que estimula el mantenimiento de la abstención o del consumo moderado. Es un elemento que evidencia el paso progresivo de procesos cognitivos y emocionales de referentes externos (perder a otros) a procesos en los que se privilegian los referentes de tipo interno y asociados al logro personal (locus de control interno). Para el caso de los hombres, aunque el temor a perder relaciones afectivas importantes sigue siendo un elemento que motiva la recuperación, su relevancia disminuye notablemente; sin embargo cabe destacar como para el caso de las mujeres, este tipo de motivaciones pierden importancias por completo y las que en su lugar aparecen se centran en análisis del tipo costo-beneficio, análisis estrictamente racionales.

Es demasiado temprano para afirmar que se encontraron los mismos resultados que en los trabajos clásicos de Sobell y sus colaboradores (1992, 1993, 1996, 2000 y 2002). Se requiere aumentar significativamente el número de sujetos y, manteniendo condiciones iguales, continuar construyendo una pirámide de información de la cual puedan desprenderse conocimientos cada vez más refinados. También es necesario profundizar sobre eventuales diferencias en la severidad del problema tal como se presenta en los dos grupos: algunos han sostenido que quienes se recuperan sin tratamiento están menos severamente afectados por el problema que quienes requieren tratamiento; los datos disponibles en esta investigación no permiten todavía aclarar este punto.

Las principales dificultades en la realización de este estudio estuvieron relacionadas con la convocatoria y consecución de las muestras inicialmente planteadas. De hecho fue por estas razones que la muestra se redujo a dos grupos (inicialmente se plantearon tres): grupo con tratamiento formal y grupo sin tratamiento formal. Las estrategias utilizadas para la convocatoria del grupo "con" tratamiento fueron relativamente sencillas, puesto que se aprovecharon las relaciones con profesionales de las adicciones y los contactos propios de Nuevos Rumbos. Sin embargo para la consecución del segundo grupo, a pesar de haberse utilizado diferentes estrategias, los resultados, después de 16 meses de convocatoria, fueron los presentados en este informe. Es necesario destacar que aunque inicialmente para el caso de la muestra de hombres se contemplaba un tercer grupo de estudio (haber acudido a algún tratamiento formal o grupo de autoayuda, o algún tipo de intervención médica en el pasado, sin lograr éxito; haber seguido consumiendo tras el tratamiento durante al menos dos años, y finalmente haberse recuperado sin tratamiento formal), las condiciones de la investigación hicieron imposible la consecución de esta muestra.

Aun con estas limitaciones, este estudio es el primero que se realiza en América Latina y tiene una muestra total superior (59 personas) a los de muchos de los estudios del mismo tipo realizados en otros países. Contrariamente a lo que los autores pensaron 
inicialmente, las personas que han pasado por estos procesos no se prestan fácilmente para hablar de ellos, y esto es particularmente cierto para quienes no han asistido a tratamientos formales. Quienes han pasado por procesos de tratamiento y han fracasado, y posteriormente se recuperaron sin ayuda, son aun más reticentes: no fue posible conseguir ni un solo caso en casi un año de búsqueda.

Los resultados obtenidos pueden ser utilizados en el contexto de los tratamientos formales con el objeto de fortalecerlos, hacerlos más eficientes y promover la sostenibilidad de los resultados.

\section{REFERENCIAS}

Bischof, G., Rumpf, H. J., Hpke, U., Meyer, C. y John, $U$. (2003). Types of natural recovery from alcohol dependence: A cluster analytic approach. Addiction, 98, 1737-46.

Burman, S. (1997). The challenge of sobriety: Natural recovery without treatment and self-help groups. Journal of Substance Abuse, 9, 41-61.

Bergin, A. (1971). The evaluation of therapeutic outcomes. En Bergin A, Garfield S, (eds.) Handbook of psychotherapy and behavior change (First edition pp. 217-270). New York: Wiley.

Cunningham, J. A. (1990). Resolving alcohol-related problems with and without treatment: the effects of different problem criteria. Journal of Studies on Alcoho, $60,463-466$.

Cunningham, J. A, Lin, E., Ross, H. E. y Walsh, G. W. (2000). Factors Associated with Untreated remission from Alcohol abuse or Dependence. Addictive Behaviors, 25, 317-321.

Finfgeld, D. L. (1997). Resolution of drinking problems without formal treatment. Perspective in Psychiatric Care, 33, 3.

Humphreys, K., Moos, R. H. y Finney, J. W. (1995). Two pathways out of drinking problems without professional treatment. Addictive Behaviors, 20, 427-441.

King, P. M. y Tucker, J. A. (2000). Behavior change patterns and strategies distinguishing moderation drinking and abstinent during the natural resolution of alcohol problems without treatment. Psychology of Addictive Behaviors, 14, 48-55.

Klingemann, H., Sobell, L. C., Baker, J., Blomqvist, J., Cloud, W., Ellinstad, T. y Finfgeld, D. (1991). The motivation for change from problem alcohol and heroin use. British Journal of Addiction, 86, 727-744.

Granfield, R. y Cloud, W. (1996). The elephant that no one sees: natural recovery among middle-class addicts. Journal of Drug Issues, 26, 45-61.

Granfield, R., Hodging, D., Hunt, G., Junker, C., Moggi, F., Peele, S., Smart, R., Sobell, M. B. y Tucker, J. (2001). Promoting Self-change from Problem Substance Use, Practical implication and treatment. Dordrecht, The Netherlands: Kluwer Academic Publishers.
Ludwig, A.M. (1985). Cognitive process associated with spontaneous recovery from alcoholism. Journal of Study on Alcohol, 46, 53-58.

Pérez, A. (2003). Instrumentos para la evaluación de procesos relacionados con recuperación natural de problemas de consumo de alcohol y otras sustancias. En Pérez, A., y Sierra, D., Informe Final sobre recuperación natural y recuperación con tratamiento del consumo de drogas y alcohol. Manuscrito no publicado, 2005. Bogotá: Universidad Católica de Colombia.

Rachman, S. (1971). The Effects of Psychoterapy. Oxford: Pergamon Press

Robins, L., Locke, B. y Regier, D. (1991). An overview of psychiatric disorders in America, en L.N. Robins y D.A. Regier (eds.), Psychiatric Disorders in America: the Epidemiologic Catchment Area Study (pp. 328-366). New York: Free Press,

Russell, M., Peirce, R.S., Chan, A.W., Wieczorek, W.F., Moscato, B.S. y Nochajski, T.H. (2001). Natural recovery in a community-based sample of alcoholics: study design and descriptive data. Substance Use and Misuse, 36, 1417-1441.

Sobell, L. (2002). Natural recovery from alcohol abuse: findings and implications for treatment and public policy. World Forum: Drugs, dependencies, impacts and responses. First International Conference on Harm Reduction and Alcohol: Towards a Comprehensive Alcohol Policy for Countries in Transition and Developing Countries. Recife, Brazil.

Sobell, L. C., Sobell, M. B. y Toneatto, T. (1992). Recovery from alcohol problems without treatment. En $\mathrm{N}$. Heather, N., W.R. Miller y J. (eds.), Self control and addictive behaviours. New York: Maxwell MacMillan.

Sobell, L. C., Sobell, M. B., Toneatto, T. y Leo, G. I. (1993). What triggers the resolution of alcohol problems without treatment?. Alcoholism: Clinical and Experimental Research, 17, 217-244.

Sobell, L. C., Sobell, M. B. y Cunningham, J. A. (1996). Recovery from alcohol problems with and without treatment: Prevalence in two population surveys. American Journal of Public Health, 86, 966-971.

Sobell, L. C., Sobell, M. B., Timothy, P. y Ellingstadt, T. P. (2000). "Natural Recovery" Meeting, Natural recovery from alcohol and drug problems: methodological review of the research with suggestions for future directions. Addiction, 95, 749-764.

Sobell, L. C., Sobell, M. B., Leo, G. I., Agrawal, S., JohnsonYoung, L. y Cunningham, J. A. (2002). Promoting self-change with alcohol abuser: A community-level mail intervention based on natural recovery studies. Alcoholism: Clinical and Experimental Research, 26, 936-948.

Slutske, W. S. (2006). Natural recovery and treatmentseeking in pathological gambling: results of two U.S. national surveys. American Journal of Psychiatry, 163, 297-302.

Waldorf, D., Reinarman, C. y Murphy, S. (1991). Cocaine changes: the experience of using and quitting. Philadelphia: Temple University Press 


\section{ANEXO 1: ENTREVISTA SEMIESTRUCTURADA}

1. ¿Cuál (es) de esta(s) sustancias consumía? (marque con una X al frente)

Otras:

\begin{tabular}{|l|l|}
\hline Alcohol & \\
\hline Marihuana & \\
\hline Cocaína & \\
\hline Heroína & \\
\hline
\end{tabular}

2. ¿Con qué frecuencia consumía cada una de estas sustancias? (leer las categorías)

\section{Alcohol}

\begin{tabular}{|l|l|}
\hline Todos los días & \\
\hline De 3 a 6 días a la semana & \\
\hline De 1 a 2 días a la semana & \\
\hline De 1 a 3 veces al mes & \\
\hline De 4 a 11 veces al año & \\
\hline Menos de 4 veces al año & \\
\hline
\end{tabular}

\section{Cocaína}

\begin{tabular}{|l|l|}
\hline Todos los días & \\
\hline De 3 a 6 días a la semana & \\
\hline De 1 a 2 días a la semana & \\
\hline De 1 a 3 veces al mes & \\
\hline De 4 a 11 veces al año & \\
\hline Menos de 4 veces al año & \\
\hline
\end{tabular}

\section{Marihuana}

\begin{tabular}{|l|l|}
\hline Todos los días & \\
\hline De 3 a 6 días a la semana & \\
\hline De 1 a 2 días a la semana & \\
\hline De 1 a 3 veces al mes & \\
\hline De 4 a 11 veces al año & \\
\hline Menos de 4 veces al año & \\
\hline
\end{tabular}

\section{Heroína}

\begin{tabular}{|l|l|}
\hline Todos los días & \\
\hline De 3 a 6 días a la semana & \\
\hline De 1 a 2 días a la semana & \\
\hline De 1 a 3 veces al mes & \\
\hline De 4 a 11 veces al año & \\
\hline Menos de 4 veces al año & \\
\hline
\end{tabular}

3. Si consumía más de una sustancia: ¿Dejó de consumirlas simultáneamente? SI ; NO

Si no las dejó simultáneamente ¿Cuál fue el orden en que dejó de consumirlas?

\begin{tabular}{|l|l|l|l|l|l|l|l|}
\hline Alcohol & & Marihuana & & Cocaína & & Heroína & \\
\hline
\end{tabular}

¿Más o menos cuánto tiempo transcurrió en ese proceso?

4. ¿Durante cuánto tiempo consumió antes de tomar la decisión de no consumir o de consumir en cantidades tan pequeñas y con tan baja frecuencia que no produjera ningún problema?

\section{A) RECUPERACIÓN}

5. ¿Cuáles fueron las personas más significativas para dejar de consumir (o para consumir en cantidades tan pequeñas y con tan baja frecuencia que no produjera ningún problema)?

Enumérelas según su grado de importancia:

¿Porqué

6. ¿Cuál fue la dificultad más grande que tuvo que afrontar para dejar de consumir (o para consumir en cantidades tan pequeñas y con tan baja frecuencia que no produjera ningún problema)? Y ¿Por qué?:

7. ¿Cuál fue el aspecto de su vida que más tuvo que modificar para poder dejar de consumir (o para consumir en cantidades tan pequeñas y con tan baja frecuencia que no produjera ningún problema)? Y ¿Por qué?:

8. ¿Cuál era el mayor temor que le producía no dejar de consumir? Y ¿Por qué?: 
9. Las preguntas que vienen a continuación, deberá responderlas haciendo referencia a las razones que usted tuvo para recuperarse.

Marque en la primera columna $V=$ verdadero o $F=$ falso; si su respuesta es ' $V$ ', marque en la segunda columna el "nivel de Importancia":

1 ("muy importante en mi decisión"), 2 ("de mediana importancia") ó 3 ("poco importante")

a. Razones para la recuperación (razones por las cuales Ud. dejó de consumir alcohol y otras sustancias, o cambió la cantidad y la frecuencia hasta lograr un consumo no problemático)

F - V importancia

a1. El consumo me estaba provocando dificultades con la ley

a2. Estaba muy preocupado por mi salud, pues ya tenía síntomas físicos inquietantes

a3. Vi que otras personas sufrían graves enfermedades por el consumo

a4. Alguien cercano falleció

a5. Me comprometí o me casé

a6. Tuve un hijo

a7. Mis condiciones de vida cambiaron (cambio de ciudad, vecindario)

a8. Conseguí mi primer trabajo

a9. Ascendí en mi trabajo o conseguí uno mejor

a10. Me estaba sintiendo incompetente en mi trabajo

a11. En el trabajo me amenazaron con despedirme

a12. Perdí mi trabajo

a13. Fracasé en mis estudios

a14. El consumo estaba afectando mi relación de pareja

a15. Rompí con mi pareja por los problemas de consumo

a16. El consumo estaba afectando mis relaciones familiares, (conflictos)

a17. Me estaba alejando de mi de mi familia

a18 El consumo estaba afectando mi vida social y amistades, (conflictos)

a19. Me estaba alejando de mis amigos

a20. Alguien cercano me dio un ultimátum para que dejara el consumo

a21. Me sentía muy avergonzado con mi comportamiento cuando consumía

a22. Me estaba sintiendo nervioso, deprimido, irritable

a23. Quería probarme a mi mismo que no era adicto

a24. Quería probarle a otras personas que no era adicto

a25. Me estaba sintiendo incongruente con mis creencias religiosas

a26. Los costos económicos del consumo eran muy altos

a27. Perdí bienes materiales por el consumo

a28. Me di cuenta de lo mal que estaban otras personas que consumían como yo lo hacía

a29. El consumo estaba teniendo efectos muy negativos en mi vida en general.

30. Empecé a sentir miedo de las consecuencias que estaba trayendo mi consumo

Describa que otras razones influyeron para que usted tomara la decisión de recuperarse: Explique cómo veía las cosas en ese momento y porqué 


\section{B) FACTORES DE MANTENIMIENTO}

10. ¿Cuáles son las personas más significativas para mantener su decisión de dejar de consumir (o de consumir en cantidades tan pequeñas y con tan baja frecuencia que no produjera ningún problema)? Enumérelas según su grado de importancia:

¿Por qué?

11. ¿Cuál es la dificultad más grande que tiene que afrontar para mantener su decisión de dejar de consumir (o de consumir en cantidades tan pequeñas y con tan baja frecuencia que no produjera ningún problema)?

Y ¿Por qué?:

12. ¿Cuál es el aspecto de su vida que más tuvo que modificar para poder mantener su decisión de dejar de consumir (o de consumir en cantidades tan pequeñas y con tan baja frecuencia que no produjera ningún problema)?

Y ¿Por qué?:

13. ¿Cuál Ud es el mayor temor que le produce la idea de no mantener su decisión de dejar de consumir (o de consumir en cantidades tan pequeñas y con tan baja frecuencia que no produjera ningún problema)?

Y ¿Por qué?:

14. Marque en la primera columna $V=$ verdadero o $F=$ falso; si su respuesta es ' $V$ ', marque en la segunda columna el "nivel de Importancia":

1 ("muy importante en mi decisión"), 2 ("de mediana importancia") ó 3 ("poco importante")

b. Factores de mantenimiento (factores que le han permitido mantener su recuperación)

\begin{tabular}{|c|c|c|}
\hline & $\mathbf{F}-\mathbf{V}$ & importancia \\
\hline \multicolumn{3}{|l|}{ b1. Dejé de tener dificultades con la ley } \\
\hline \multicolumn{3}{|l|}{ b2. Mi salud empezó a mejorar y me recuperé físicamente } \\
\hline \multicolumn{3}{|l|}{ b3. Cambié de hábitos alimenticios, mejoré la alimentación } \\
\hline \multicolumn{3}{|l|}{ b4. Empecé a hacer ejercicio } \\
\hline \multicolumn{3}{|l|}{ b5. Aumenté mi rutina de ejercicios } \\
\hline \multicolumn{3}{|l|}{ b6. Encontré un trabajo que me estimulaba y me absorbía positivamente } \\
\hline \multicolumn{3}{|l|}{ b7. Mis finanzas mejoraron notablemente } \\
\hline \multicolumn{3}{|l|}{ b8. Persuadirme para ir a trabajar } \\
\hline \multicolumn{3}{|l|}{ b9. Persuadirme ir a estudiar } \\
\hline \multicolumn{3}{|l|}{ b10. Ver logros en mis estudios } \\
\hline \multicolumn{3}{|l|}{ b11. La religión ha sido un soporte permanente } \\
\hline \multicolumn{3}{|c|}{ b12. Me interesé por situaciones y actividades (cotidianas o de ocio) en las que el consumo no tiene cabida } \\
\hline \multicolumn{3}{|c|}{ b13. Le di un giro total a mi vida: cambié de costumbres, de metas y de intereses } \\
\hline \multicolumn{3}{|l|}{ b14. Recibir apoyo de mi pareja } \\
\hline \multicolumn{3}{|l|}{ b15. La disminución de conflictos con mi pareja } \\
\hline \multicolumn{3}{|l|}{ b16. Recibir apoyo de mi familia } \\
\hline \multicolumn{3}{|l|}{ b17. La disminución de conflictos con mi familia } \\
\hline \multicolumn{3}{|l|}{ b18. Recibir apoyo de mis amigos } \\
\hline \multicolumn{3}{|l|}{ b19. Cambié completamente de amigos, los nuevos no eran consumidores } \\
\hline \multicolumn{3}{|l|}{ b20. Entré en contacto con personas que dejaron de consumir } \\
\hline \multicolumn{3}{|l|}{ b21. Evito todas las situaciones en las que hubiera riesgo de consumo } \\
\hline \multicolumn{3}{|l|}{ b22. Pensar en la gente que me ha ayudado } \\
\hline \multicolumn{3}{|l|}{ b23. Pensar en las promesas que les he hecho a otros } \\
\hline \multicolumn{3}{|l|}{ b24. Pensar en las promesas que me he hecho a sí mismo } \\
\hline b25. Pensar en los problemas que el consumo me generó en el pasado & & \\
\hline
\end{tabular}




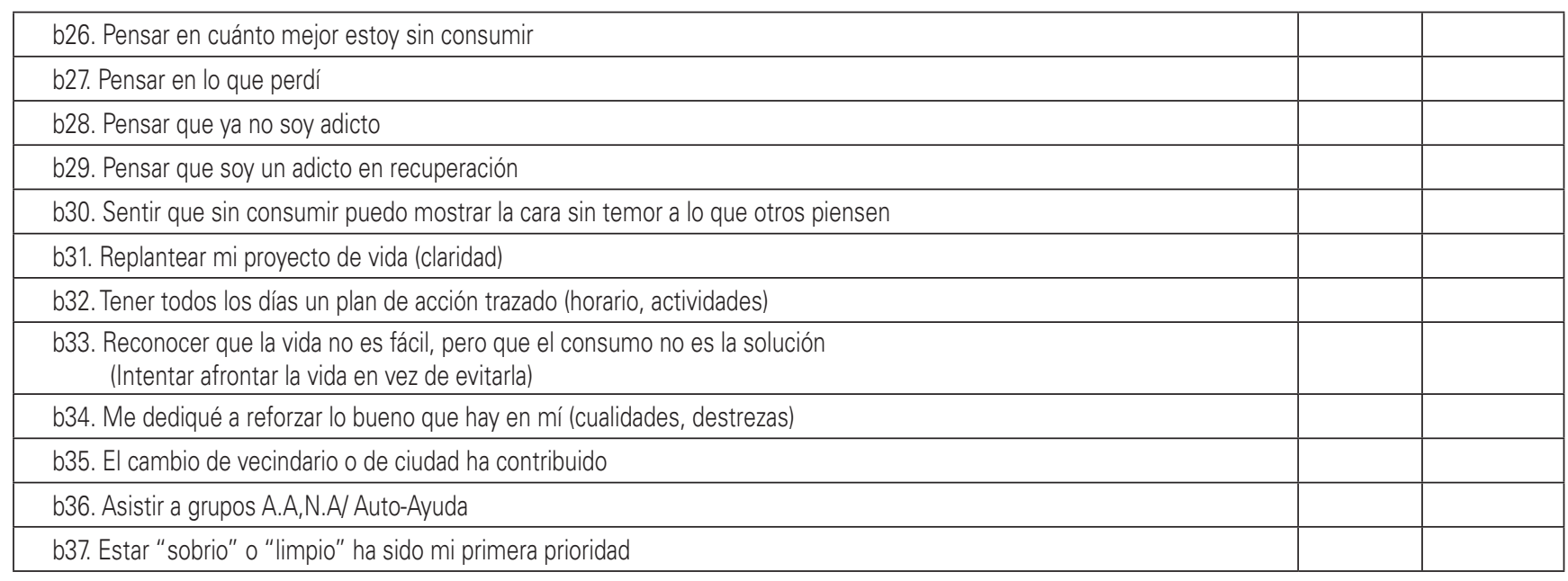

Describa qué otros factores han influido para mantener su recuperación:

¿Por qué?

\section{C) OBSTÁCULOS PARA NO ASISTIR A TRATAMIENTO Y RAZONES PARA ABANDONARLO}

15. Marque en la primera columna $V=$ verdadero o $F=$ falso; si su respuesta es ' $V$ ', marque en la segunda columna el "nivel de Importancia":

1 ("muy importante en mi decisión"), 2 ("de mediana importancia") ó 3 ("poco importante")

\section{Obstáculos para asistir a tratamiento}

\begin{tabular}{|l|c|c|}
\hline \multicolumn{1}{l}{ F - V } & importancia \\
\hline c2. Pensaba que mi problema no era tan grave como para ir a tratamiento & & \\
\hline c3. Sentía vergüenza de imaginarme en tratamiento & & \\
\hline c4. Lo que había oído me parecía inaceptable & & \\
\hline c5. Los tratamientos existentes no son buenos & & \\
\hline c6. Eso hace parte de mi intimidad, no quería hablarlo con nadie & & \\
\hline c7. Me preocupaba que me señalaran como "loco". & & \\
\hline c9. No quería que me rotularan de "adicto" & & \\
\hline
\end{tabular}

Describa qué otros obstáculos hicieron que usted no asistiera a tratamiento:

\section{D) Razones para abandonar el tratamiento}

\begin{tabular}{|l|c|c|}
\multicolumn{2}{|l}{ F - V } & importancia \\
\hline d1. Era muy costoso & & \\
\hline d2. Mi problema no era tan grave para continuar el tratamiento & & \\
\hline d3. Sentía que el tratamiento no me servía para nada & & \\
\hline d4. No quería seguir pensando que era un adicto & & \\
\hline d5. Pensaba que mi adicción no era "un viaje por toda la vida" & & \\
\hline d6. No veía cambios en mi sistema familiar & & \\
\hline d7. No quería ser abstemio & & \\
\hline d8. Me daba pereza esforzarme y no quería hacer cambios radicales en mi estilo de vida & & \\
\hline
\end{tabular}

Describa qué otras razones hicieron que usted abandonara el tratamiento 


\section{ANEXO 2: RAZONES PARA LA RECUPERACIÓN, FACTORES DE MANTENIMIENTO Y OBSTÁCULOS PARA ASISTIR A TRATAMIENTO}

Ud. encontrará a continuación tres grupos de preguntas: el primero se refiere a las razones por las cuales Ud. dejó de consumir alcohol y otras sustancias, o cambió la cantidad y la frecuencia hasta lograr un consumo no problemático; el segundo se refiere a los factores que le han permitido mantener su recuperación; y el tercero a las razones que $\mathrm{Ud}$. tuvo para no asistir a un tratamiento, o para abandonarlo si lo inició. En la primera columna $U d$. deberá marcar $V=$ verdadero o $F=$ falso; cuando su respuesta sea ' $V$ ', en la segunda columna, "Importancia", Ud. deberá marcar 1 ("muy importante en mi decisión"), 2 ("de mediana importancia") ó 3 ("poco importante").

\section{A. Razones para la recuperación}

\begin{tabular}{|c|c|c|}
\hline & $\mathbf{F}-\mathbf{V}$ & importancia \\
\hline 1. Me estaba provocando dificultades con la ley. & & \\
\hline 2. Tomé decisiones importantes que afectaban mi estilo de vida (casarse, tener un hijo). & & \\
\hline 3. Empecé a tener mucho miedo a las consecuencias de lo que estaba haciendo. & & \\
\hline 4. Empecé a pensar sobre el consumo de una manera diferente: me parecía absurdo e in & & \\
\hline 5. El consumo estaba dañando mis relaciones afectivas. & & \\
\hline 6. Mis condiciones de vida cambiaron (cambio de ciudad, cambio de vecindario o de activ & & \\
\hline 7. El consumo estaba afectando mi vida social y de amistades. & & \\
\hline 8. Me estaba sintiendo muy preocupado(a) por mi salud, pues tenía síntomas inquietante & & \\
\hline 9. Me estaba sintiendo incongruente con mis creencias religiosas. & & \\
\hline 10. El consumo me había llevado a ser incompetente en mi trabajo (o me amenazaron col & & \\
\hline 11. El consumo estaba afectando mis relaciones con mi familia. & & \\
\hline 12. El consumo estaba teniendo efectos muy negativos en mi vida en general. & & \\
\hline 13. Los costos económicos del consumo eran muy altos. & & \\
\hline 14. Me di cuenta de lo mal que estaban otras personas que consumían como yo lo hacía. & & \\
\hline
\end{tabular}

B. Factores de mantenimiento

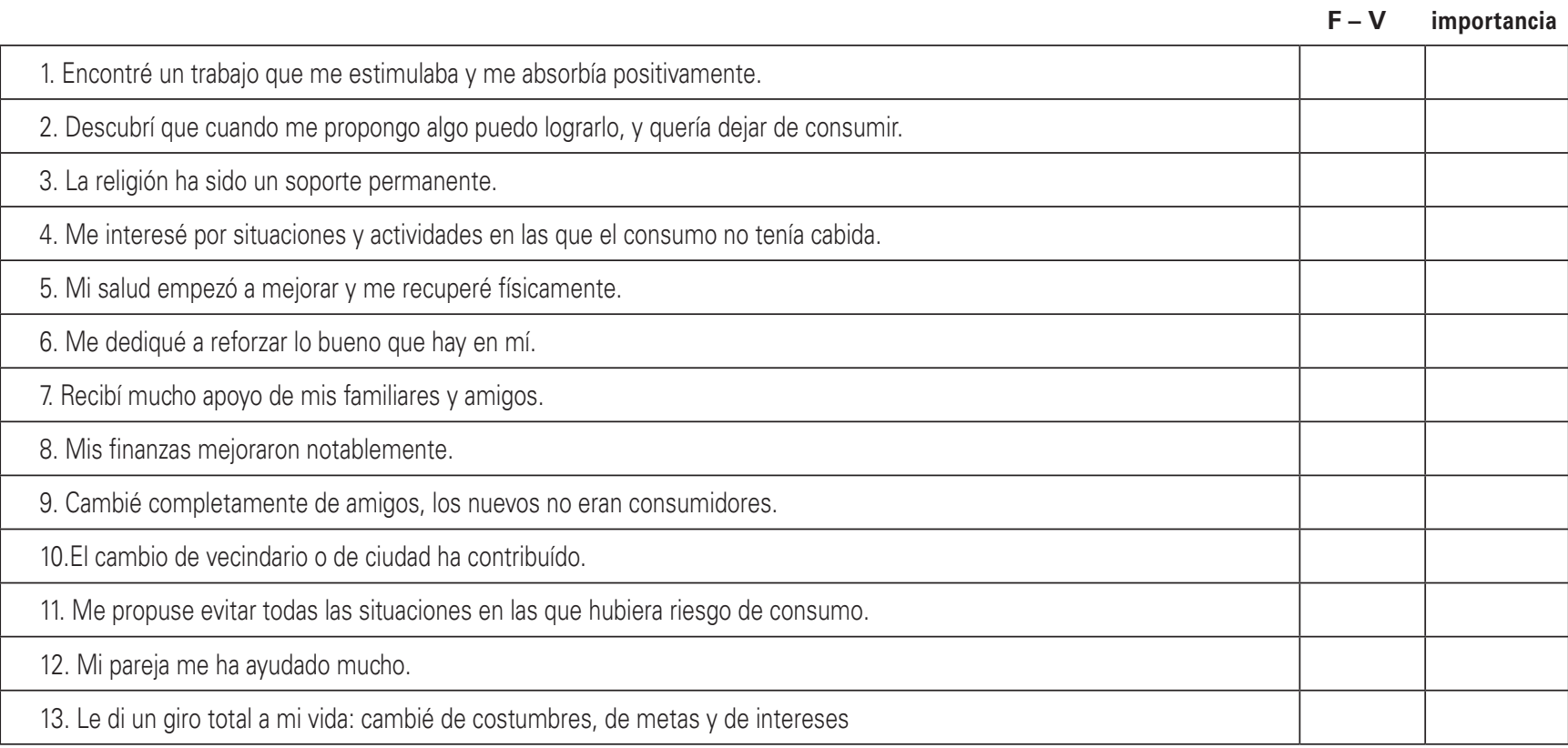




\section{Obstáculos para asistir a tratamiento}

F - V importancia

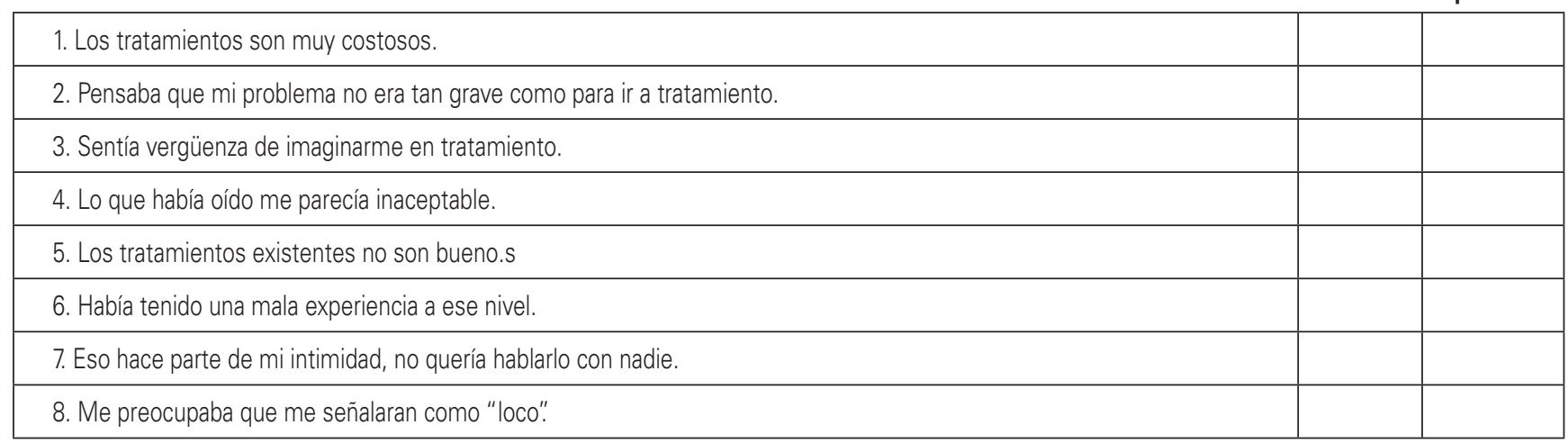


